\title{
Synthesis and electromagnetic absorption properties of micro-nano nickel powders prepared with liquid phase reduction method
}

\author{
Ying Yu*, Hua Ma**, Xiao-Xia Tian*, Hong-Liang $\mathrm{Du}^{*}$, Song $\mathrm{Xia}^{\dagger}$ and Shao-Bo $\mathrm{Qu}^{*}$ \\ *School of Science, Air Force Engineering University, Xi'an 710051, P. R. China \\ ${ }^{\dagger}$ Key Laboratory of Electronic Materials Research of Ministry of Education \\ Xi'an Jiaotong University, Xi'an 710049, P. R. China \\ tmahuar@163.com
}

Received 7 April 2016; Revised 7 August 2016; Accepted 8 August 2016; Published 21 September 2016

\begin{abstract}
Monodisperse micro-nano nickel powders have been prepared by chemical reduction of aqueous solution $\mathrm{NiSO}_{4}, \mathrm{NaOH}$ and $\mathrm{NaH}_{2} \mathrm{PO}_{2}$, and the influence of $\mathrm{pH}$ value and initial concentration of $\mathrm{NiSO}_{4}$ on the size, structure, morphology and microwave absorption properties of nickel powders were investigated. The crystal structure of nickel powders was characterized by $\mathrm{X}$-ray diffraction (XRD). And the morphology of the as-synthesized products was characterized by scanning electron microscopy (SEM) and transmission electron microscopy (TEM). The microwave absorption properties of the composite materials were characterized by network analyzer. The result indicates that the growth of nickel powders produced by $\mathrm{NiSO}_{4}$ and $\mathrm{NaH}_{2} \mathrm{PO}_{2}$ at alkaline condition deeply relies on $\mathrm{pH}$ value and initial concentration of $\mathrm{NiSO}_{4}$ in reaction system. Different sizes of nickel powders with the diameter of $1.5 \mu \mathrm{m}$ and $180 \mathrm{~nm}$ were produced at the $\mathrm{pH}$ value of 10 and initial concentration of $\mathrm{NiSO}_{4}$ at $0.5 \mathrm{~mol} /$ L. The network analyzer showed definite microwave absorption properties of nickel powders with different sizes in the range of $0.5-18.0 \mathrm{GHz}$.
\end{abstract}

Keywords: Micro-nano nickel powders; monodisperse; size; absorption; liquid phase reduction method.

\section{Introduction}

Development of electronic technology has become a more and more important role in the process of modern information field. While providing convenience for people to transfer information, it also brings a lot of electromagnetic pollution, especially the rapid development of wireless communication technology and high-frequency electronic devices in the microwave band. In order to eliminate the damage of worsening electromagnetic interference, absorbing materials are extensively used. ${ }^{1-4}$ The iron, cobalt, nickel and other magnetic materials have high magnetic permeability, large dielectric constant, strong electromagnetic loss and other characteristics. They are widely used as conventional absorbing materials. In practice, the nickel has attracted particular attention with a better corrosion resistance than iron. In recent years, researchers have dedicated to fabricating broadband, high absorption and low density electromagnetic absorbing materials. Micro-nano monodisperse spherical magnetic materials make it possible to absorb electromagnetic wave in high frequency band, since their less aggregation, large specific surface area, high saturation magnetization and large anisotropy field. Therefore, exploring a simple and enforceable synthesis in fabricating monodisperse magnetic nickel powders is necessary.

Currently, there are many methods to prepare ultrafine nickel powders, like mechanical milling method, hydrothermal method, liquid-phase reduction method and so on.
Li et al. ${ }^{5}$ prepared flake nickel powders with high energy ball milling, which have high surface finish and good dispersion, but the experimental period is too long. Wang et al. ${ }^{6}$ used autocontinuous wire electrical explosion method to fabricate nickel powders of different sizes by changing initial charging voltage. The operation of this method is simple, but it has high dependence on complex equipment. Lei et al. ${ }^{7}$ produced smooth nickel powders by liquid phase reduction method, with hydrazine as a reducing agent. But the size of prepared nickel powders is large and inhomogeneous. Based on liquid phase reduction method, ${ }^{8}$ we produced micro-nano nickel powders of different sizes, with nickel sulfate and sodium hydroxides as source materials, and sodium hypophosphite as the reducing agent. From this experiment, we obtained a simple method in controlling the size of nickel powders by changing $\mathrm{pH}$ values and initial concentrations of nickel sulfate, and verified the advantage of the acetyl acetone as a surfactant in controlling the morphology of monodisperse nickel powders. Meanwhile, we investigated the influence of the size of nickel on electromagnetic absorbing properties.

\section{Experiments}

\subsection{Materials}

Six sodium sulfate $\left(\mathrm{NiSO}_{4} \cdot 6 \mathrm{H}_{2} \mathrm{O}\right)$, sodium hydroxide $(\mathrm{NaOH})$, hypophosphite $\left(\mathrm{NaH}_{2} \mathrm{PO}_{2}\right)$, acetyl acetone, and

This is an Open Access article published by World Scientific Publishing Company. It is distributed under the terms of the Creative Commons Attribution 4.0 (CC-BY) License. Further distribution of this work is permitted, provided the original work is properly cited. 
ethanol were purchased from Tianjin Hengxing Chemical Reagent Company. All the chemical reagents used in this work were analytically pure without further purification.

\subsection{Synthesis of micro-nano nickel powders}

The micro-nano nickel powders were fabricated by liquidphase reduction method. Briefly, appropriate $\mathrm{NaOH}$ and $\mathrm{NiSO}_{4} \cdot 6 \mathrm{H}_{2} \mathrm{O}$ were first dissolved in water separately. Taking an $80^{\circ} \mathrm{C}$ water bath for $10 \mathrm{~min}$ and mixing two solutions via magnetic stirring device until formatting green colloid, and then surfactant of acetyl acetone was added. After the mixed solution was stirred evenly, we added the right amount of $\mathrm{NaH}_{2} \mathrm{PO}_{2}$ with 10 min water bath in $80^{\circ} \mathrm{C}$. Stirring constantly until generating lots of bubbles in the reaction and appearance of black precipitates indicates that nickel powders has formed. After the reaction, we used distilled water and ethanol to wash them repeatedly, and finally placed them in a vacuum oven at $80^{\circ} \mathrm{C}$ for $3 \mathrm{~h}$.

\subsection{Characterizations}

A Quanta F250 environmental scanning electron microscope (SEM) was used to observe the morphology and the size of nickel powders. The inner morphology features of nickel powders were further characterized by HITACHI HT7700 transmission electron microscopy (TEM). The crystal structure was taken on a MiniFlex600 X-ray diffraction (XRD) with a scanning range from $20^{\circ}$ to $70^{\circ}$ at a $40 \mathrm{KV}$ and $40 \mathrm{~mA}$ of $\mathrm{CuK} \alpha$. The coaxial method was used to measure the electromagnetic parameters. Firstly, paraffin and nickel powders with a mass ratio of 2:3 were weighed and mixed evenly into n-hexane under ultrasonic shocking. Then, the mixture was poured into a mold with outer diameter of $7 \mathrm{~mm}$ and inner diameter of $3 \mathrm{~mm}$ to get coaxial sample loops with a thickness of $2-3 \mathrm{~mm}$. At last, the electromagnetic parameters were tested on Agilent-N5242A vector network analyzer in the range of $0.5-18 \mathrm{GHz}$.

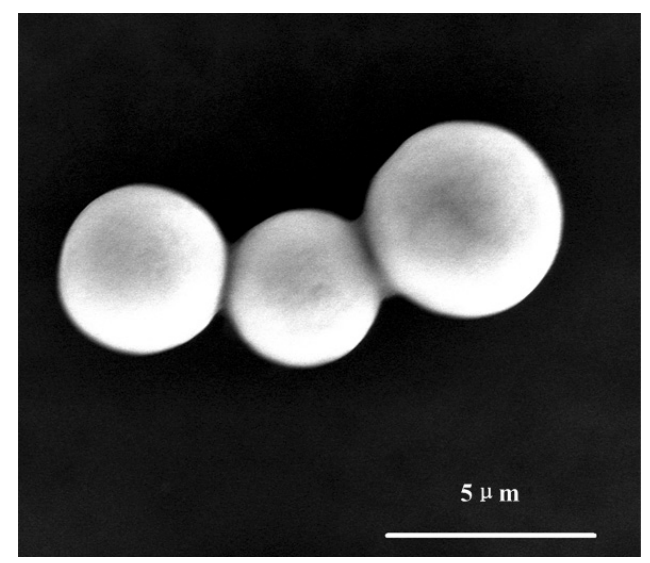

(a)

\subsection{Calculation of absorbing properties of the samples}

According to transmission line theory, ${ }^{9-12}$ the input impedance $\left(Z_{\text {in }}\right)$ of absorber depends on the characteristic impedance and the terminal impendence of materials. For a single layer of absorber with metal as the substrate, the characteristic impedence is determined by the equivalent electromagnetic parameters of its material since the conductivity of ideal metal tends to infinity. From this, the input $Z_{\text {in }}$ can be calculated by Eq. (1).

$$
Z_{\text {in }}=\sqrt{\frac{\mu_{r}}{\varepsilon_{r}}} \tanh \left[j \frac{2 \pi}{c} \sqrt{\mu_{r} \varepsilon_{r}} f d\right],
$$

where $c$ represents the velocity of electromagnetic wave in free space, $f$ is the frequency of the electromagnetic wave, $d$ is the coating thickness of the absorber, $\varepsilon_{r}$ and $\mu_{r}$ represent the complex permittivity and permeability of the composite, respectively. While giving the coating thickness and the test frequency, the reflection loss (RL) can be calculated as follows:

$$
\operatorname{RL}(d B)=20 \lg \left|\frac{Z_{\text {in }}-1}{Z_{\text {in }}+1}\right| .
$$

\section{Result and Discussion}

\subsection{The analysis on size and morphology of nickel powders produced with different $\mathrm{pH}$ values and initial concentration of nickel sulfate}

We prepared nickel powders with different sizes, which were controlled by $\mathrm{pH}$ values and initial conditions, as shown in Fig. 1. Figures 1(a)-1(c) show micro nickel powders with the diameter of $3,1.5$ and $2.5 \mu \mathrm{m}$, respectively, corresponding to the prepared conditions at $\mathrm{pH}$ value of 9,10 and 11 . It shows that the size of nickel powders is associated with $\mathrm{pH}$ value and can be controlled by $\mathrm{pH}$ value. After several comparative experiments, we have found that the $\mathrm{pH}$ value of 10 is the critical value. When the $\mathrm{pH}$ value is lower than 10 , the size of

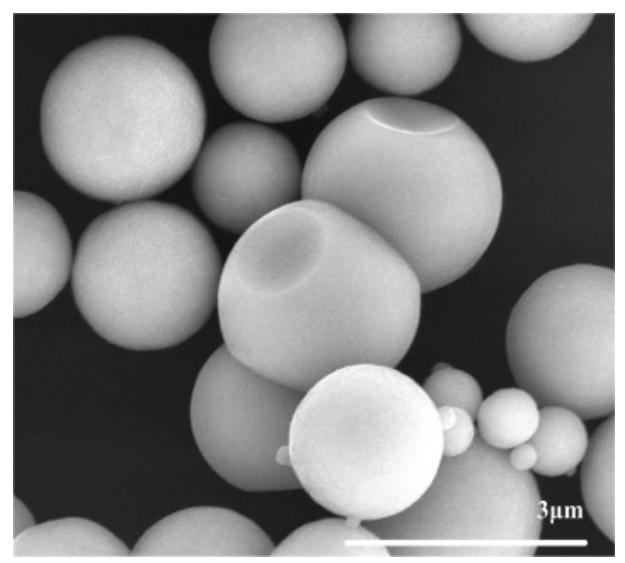

(b)

Fig. 1. SEM images of nickel powders produced with different $\mathrm{pH}$ values (a), (b), (c) and initial concentrations of $\mathrm{NiSO}_{4}(\mathrm{~d}),(\mathrm{e}),(\mathrm{f})$. 


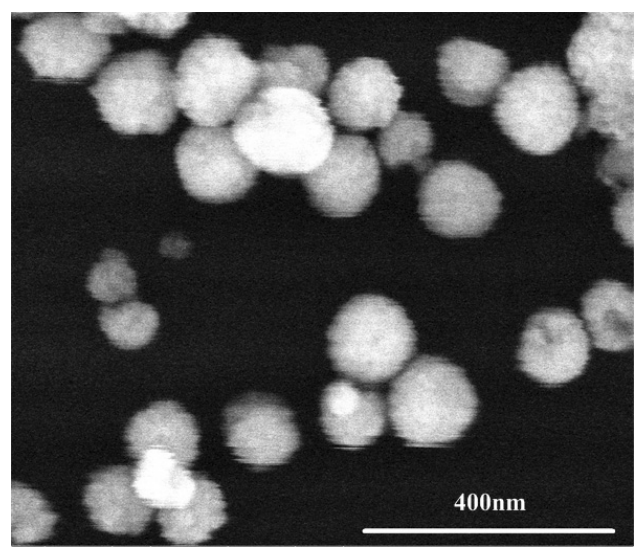

(c)

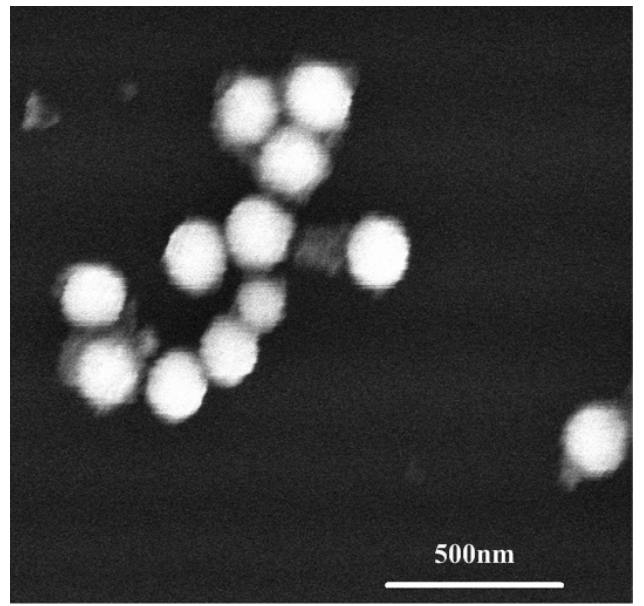

(e)

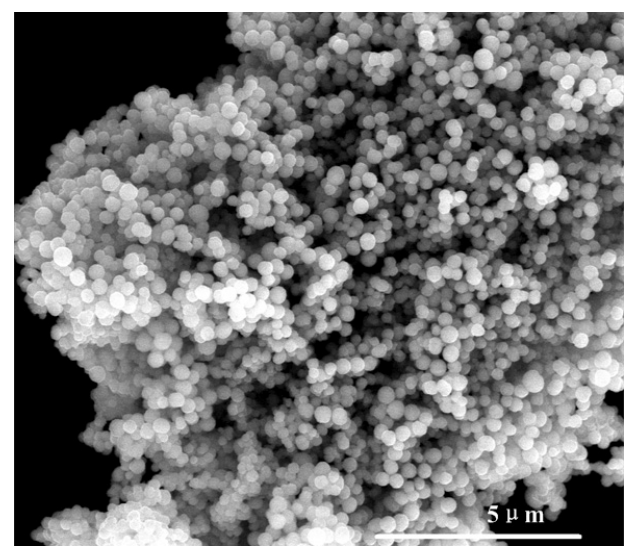

(d)

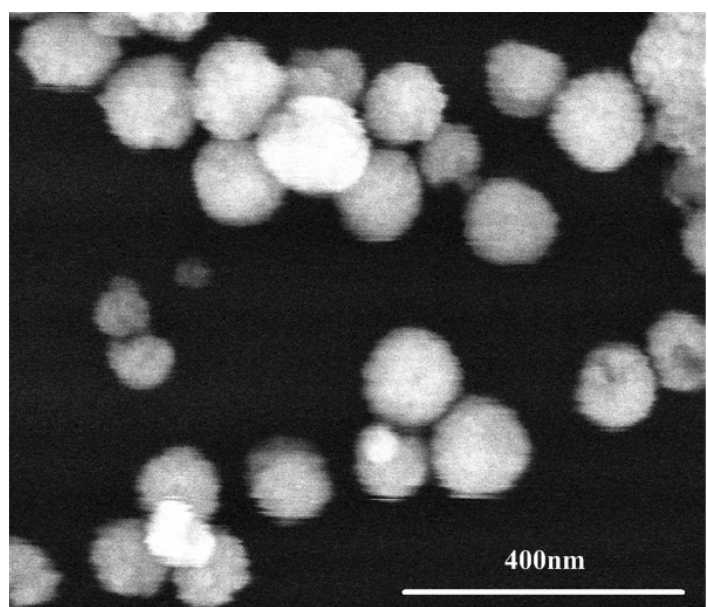

(f)

Fig. 1. (Continued)

nickel powders decreases with increasing $\mathrm{pH}$ value. While the $\mathrm{pH}$ value is higher than 10 , the size of nickel powders increases with increasing $\mathrm{pH}$ value. Therefore, $\mathrm{pH}$ value of 10 is the optimum $\mathrm{pH}$ value to fabricate the nickel powders with smallest size when other conditions remain invariable in this experiment. Figures 1(d)-1(f) show nickel powders prepared at $\mathrm{pH}$ value of 10 , with initial concentration of nickel sulfate at $0.25,0.5$ and $0.75 \mathrm{~mol} / \mathrm{L}$, respectively. The results show that when the $\mathrm{pH}$ value is constant, the size of nickel powders decreases with increasing initial concentration of nickel sulfate. Especially when the initial concentration of nickel sulfate is about $0.5 \mathrm{~mol} /$ L (Fig. 1(e)), the prepared nickel powders have smooth surface, great dispersion and uniform size. Hence, the size and the morphology of nickel powders can be controlled by the $\mathrm{pH}$ value and initial concentration of nickel sulfate.

To further investigate the influence of $\mathrm{pH}$ value and initial concentration of nickel sulfate on the structure of nickel powders, we select nickel powders under the optimum $\mathrm{pH}$
$(\mathrm{pH}=10)$ and initial concentration $(0.5 \mathrm{~mol} / \mathrm{L})$ of nickel sulfate and observed them by TEM. Figure 2(a) shows the micro nickel powders prepared at $\mathrm{pH}$ value of 10 . The micro nickel powders were made of black interior and a gray thin surface layer. The figure shows that the inside density of nickel powers is larger than that of surface. Figure 2(b) shows the nanoparticles of nickel prepared with that of the initial concentration of nickel sulfate at $0.5 \mathrm{~mol} / \mathrm{L}$. We find that both the porous structure of nickel powders from the lighter surrounding and the density of nickel powders is relatively small.

\subsection{Analysis of crystal structures of nickel powders with different sizes}

We analyze the crystal structure of macro-nano nickel powders. The curve $a$ in Fig. 3 presents the XRD pattern of nickel powders with $1.5 \mu \mathrm{m}$ prepared at $\mathrm{pH}$ value of 10 . The curve 


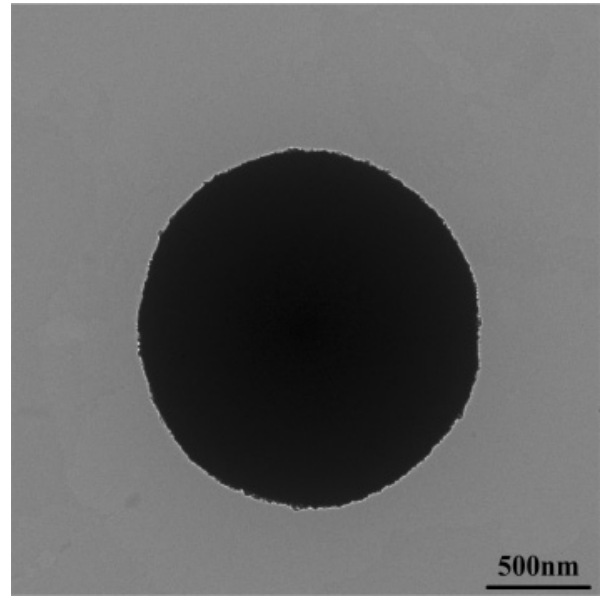

(a)

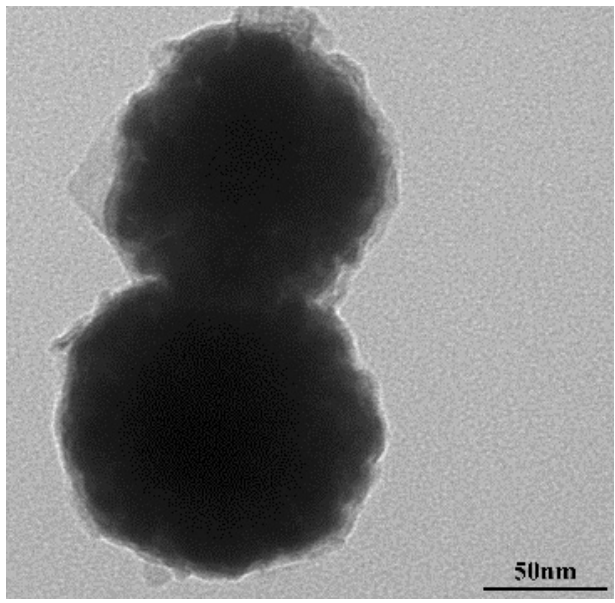

(b)

Fig. 2. TEM images of nickel powders with size of $1.5 \mu \mathrm{m}$ (a) and $180 \mathrm{~nm}(\mathrm{~b})$.

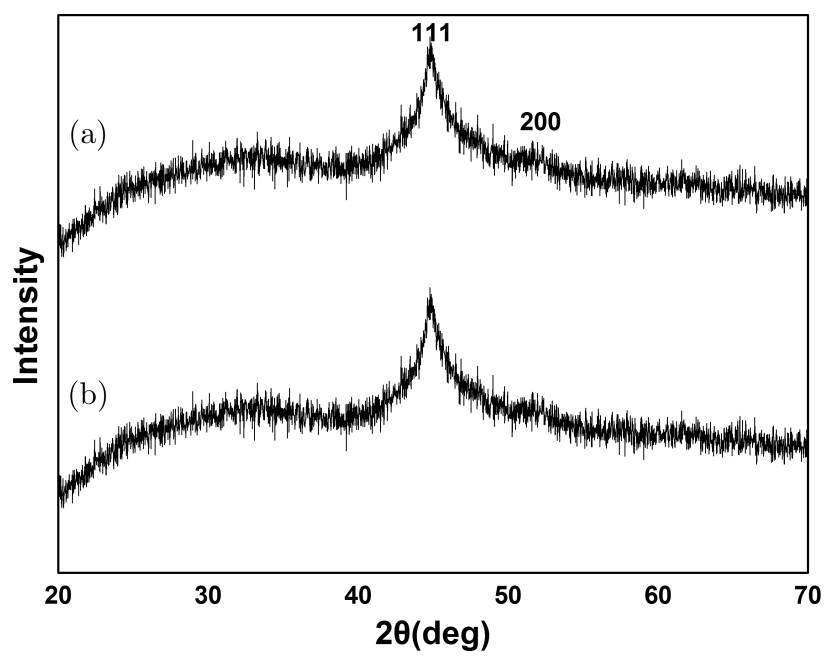

Fig. 3. XRD patterns of nickel powders with different sizes: $1.5 \mu \mathrm{m}$ (a) and $180 \mathrm{~nm}$ (b). $b$ in Fig. 3 presents the XRD pattern of nickel powders with diameter of $180 \mathrm{~nm}$ which were prepared with the initial concentration of nickel sulfate at $0.5 \mathrm{~mol} / \mathrm{L}$. Obviously, the diffraction peaks at nearly $44^{\circ}$ and $51^{\circ}$ can be easily indexed to (111) and (200) peaks of nickel (JCPDS Card No. 652865), respectively, showing that the diffraction peaks of nickel powders with different sizes approach closely and the structure of the powders is face-centered cubic crystal of nickel. Compared with the standard card, the position of diffraction peaks occurred slightly offset, and the diffraction peaks broaden and diffuse slightly. To be noted that the diffraction peak of crystal face (200) is not obvious, which may be explained that the nickel powders are too small and the crystalline is poor.

\subsection{Electromagnetic properties of nickel powders with different sizes}

In order to investigate the electromagnetic properties of nickel powders with different sizes, the electromagnetic parameters of micro-size, nano-size and mixed-size nickel powders were tested in the frequency range of $0.5-18.0 \mathrm{GHz}$. Four coaxial samples with the mass ratios of micro and nano nickel powders, 1:0, 0:1, 1:1 and 1:2, were prepared, respectively.

Then, the electromagnetic parameters of samples were tested so as to compare the difference in properties. The curves in Figs. 4(a) and 4(b) show the real part and imaginary part of permittivity of nickel powders with different sizes. We can see that in range of $0.5-18.0 \mathrm{GHz}$, the real part of permittivity of mixed nickel powders with mass ratio $1: 2$ is higher than other mixed powders, whereas the imaginary part of permittivity of all mixed powders are almost equal, except for the case of an obvious resonance on the curve of mixed nickel powders with mass ratio 1:0. The curves in Figs. 4(c) and 4(d) show the permeability of mixed nickel powders at different mass ratios, indicating that different samples have the same variation except for the case of mass ratio 1:0 at its resonance point.

To be noted that, the imaginary part of permeability is negative at some frequencies, as shown in Fig. 4(d). The first possibility may be Fabry-Perot resonance ${ }^{13}$ related to the test system. We adopted the method of the paper ${ }^{13}$ and exclude this possibility successfully by calculation and comparison. The second possibility may be electronic-magnetic energy conversion effect. ${ }^{14,15}$ As shown in Fig. 4(b), at the position of $13.0 \mathrm{GHz}$, the large imaginary part of permittivity corresponds to strong loss of energy. In contrast, as shown in Fig. 4(d), at the same position of $13.0 \mathrm{GHz}$, the imaginary part of permeability is negative and has large absolute value, which corresponds to the increase in magnetic energy. Connection with the loss curves shown in Fig. 5(a), the increase and decrease of energy can offset each other, and the total loss is larger than the gain, which does not violate the law of conservation of energy from the perspective of the 


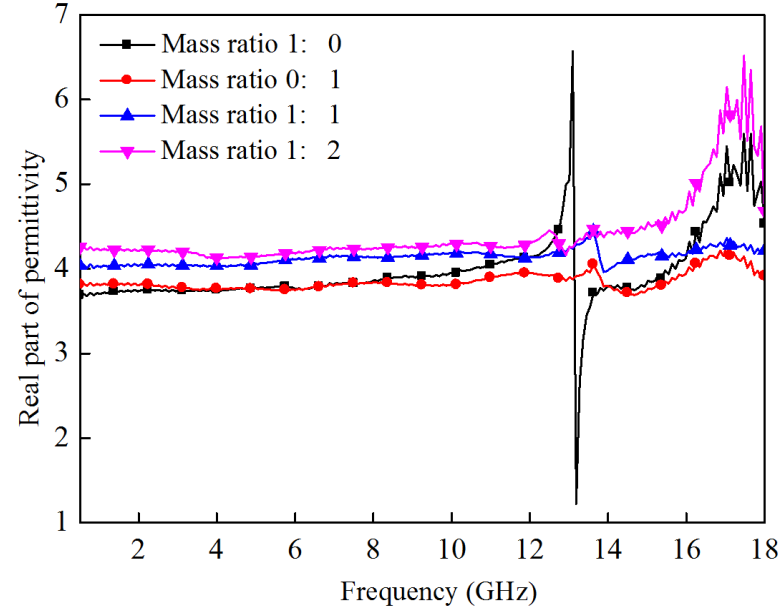

(a)

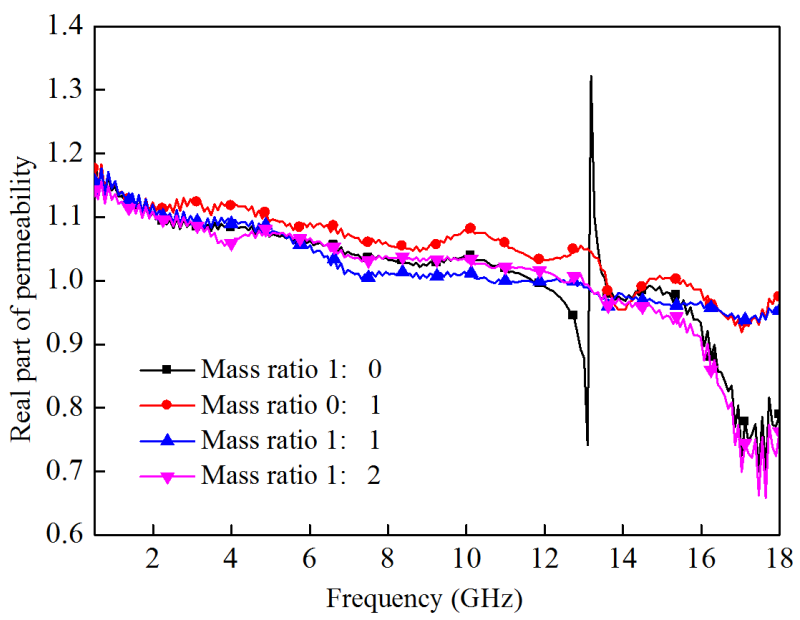

(c)

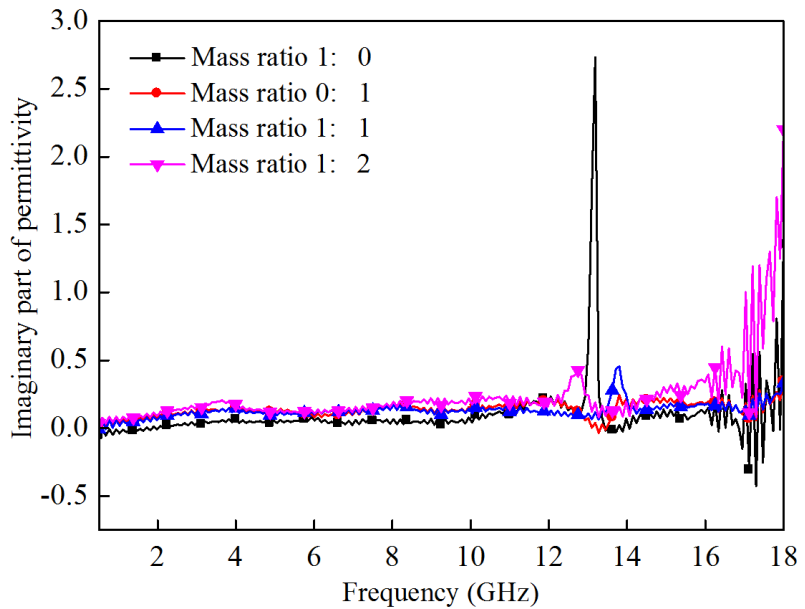

(b)

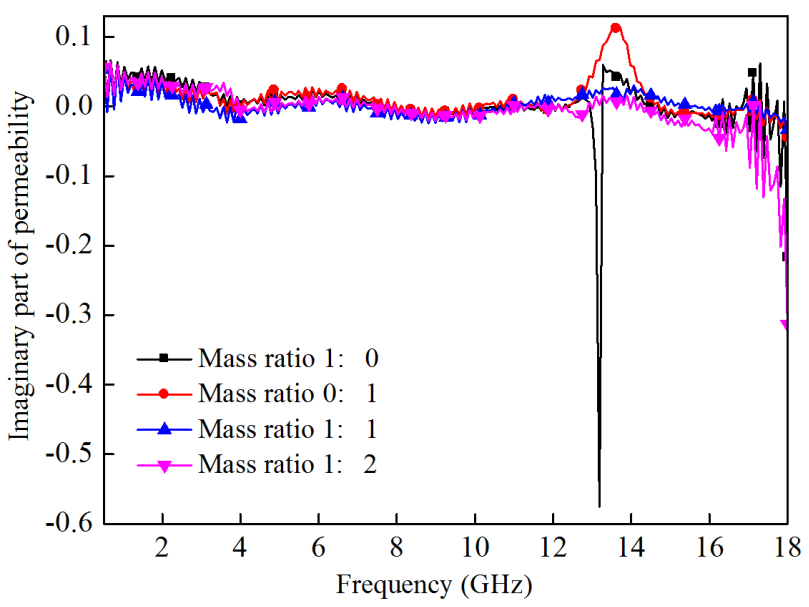

(d)

Fig. 4. The permittivity (a), (b) and permeability (c), (d) of nickel powders with different sizes as a function of frequencies.

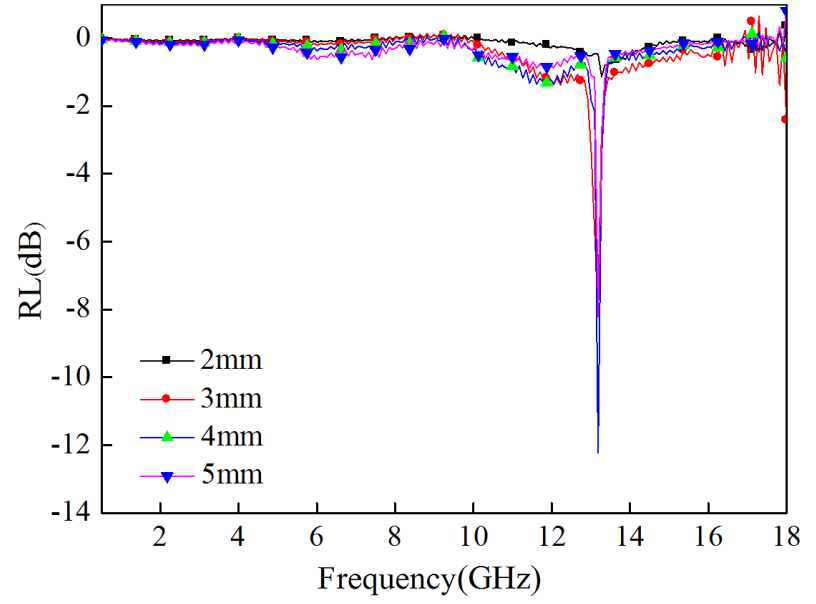

(a)

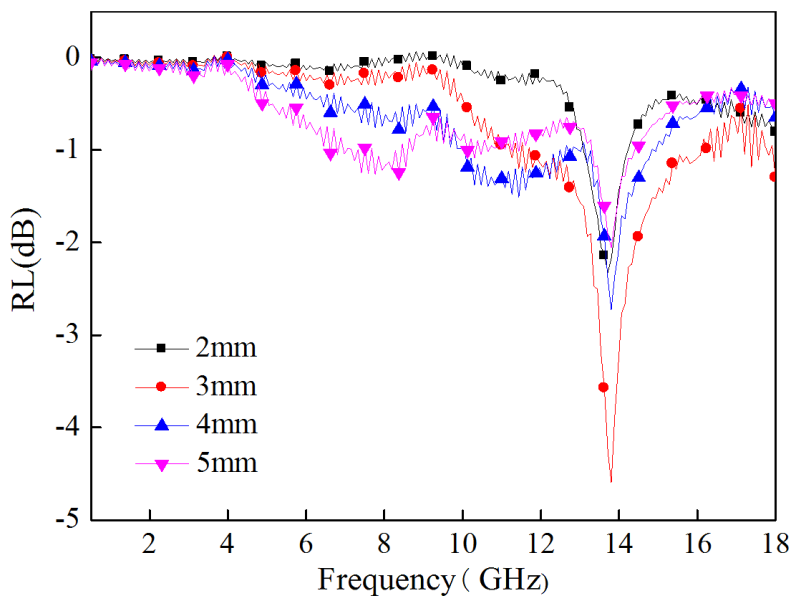

(b)

Fig. 5. RL of nickel powders with different micro-nano mass ratios 1:0 (a), 0:1 (b), 1:1 (c), 1:2 (d) at different thicknesses. 


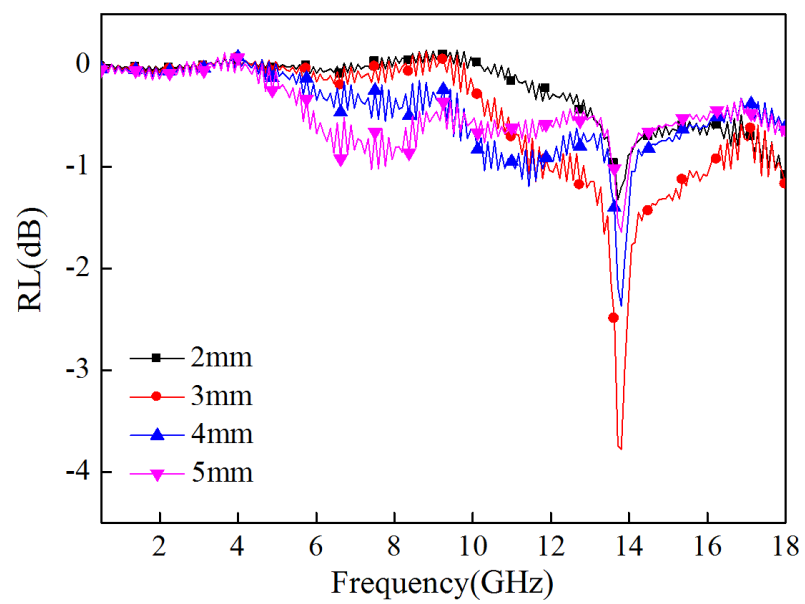

(c)



(d)

Fig. 5. (Continued)

electromagnetic energy. Therefore, the negative imaginary part of permeability in the resonance point obtained from test is physically reasonable.

\subsection{Absorption properties of nickel powders with different sizes}

The absorption properties of micro-nano materials are related to their type, morphology, size and skin depth. Here, we mainly analyze the effects of size and skin depth on absorption properties. The reflect loss curves in Figs. 5(a)-5(d) correspond to measured results of the mixed samples with micro and nano nickel powders with mass ratios of 1:0, 0:1, $1: 1$ and $1: 2$, respectively.

It shows that the nickel powders with mass ratio of 1:0 have the highest absorptivity at the thickness of $5 \mathrm{~mm}$, with taking an optimal RL value of $-12.2 \mathrm{~dB}$ and a narrow bandwidth. The RL value of nickel powders with mass ratio $0: 1$ approaches $-5 \mathrm{~dB}$ in the resonance point at the thickness of $3 \mathrm{~mm}$, for which the absorptivity of the sample is lower than that of the case with mass ratio 1:0, but it has a wide bandwidth. The absorptivity of the nickel powders with mass ratios 1:1 and 1:2 are not as good as a single micro or nanometer nickel powders. Comparing these four cases, we find that the thickness of sample has an effect on the absorption of materials, but not the thicker, the better. As shown in Figs. 5(b)-5(d), the absorption of samples at thickness of $3 \mathrm{~mm}$ is superior to others, which may be explained that the size of micro nickel powders is close to the skin depth and results in more extensive absorbing mechanism. Therefore, adopting the liquid phase reduction method to control the size of nickel powders, the absorber composed by micro-nano nickel powders can be designed and fabricated to realize absorption efficiency.

\section{Conclusion}

Using liquid phase reduction method, we prepared nickel powders, the size and morphology of which were controlled by changing $\mathrm{pH}$ values and initial concentrations of nickel sulfate. The effect of $\mathrm{pH}$ value on the size is that $\mathrm{pH}$ value has a critical value, for example 10 in our case, and taking this value as critical point, the size of nickel powders increases firstly and then decreases with increasing $\mathrm{pH}$ values. The effect of initial concentration of nickel sulfate on the size is that within a certain range, the size of nickel powders becomes smaller with increase of initial concentrations of nickel sulfate. We also found that, the microwave absorption of nickel mixture is related to the size of nickel powders, and particularly has better efficiency when the size is close to the skin depth of materials. Therefore, designing and preparing nickel powders with their size and morphology being controlled by changing $\mathrm{pH}$ values and initial concentrations of nickel sulfate has a nice prospect in the field of electromagnetic absorbers.

\section{Acknowledgments}

This research is supported by the National Natural Science Foundation of China under Grant No. 61331005, the National Natural Science Foundation of China under Grant No. 11274389, the National Natural Science Foundation of China under Grant No. 21471159, the Special Funds for Author of Annual Excellent Doctoral Degree Dissertation of China under Grant No. 201242, the fund of the State Key Laboratory of Inorganic Synthesis and Preparative Chemistry under Grant No. 2015-09, the Shaanxi Provincial Natural Science Foundation of China under Grant No. 2015JM2042 and the Shaanxi Province Scientific and Technology 
Innovation Team Foundation of China under Grant No. 2014KCT-05.

\section{References}

${ }^{1}$ K. Matsumoto, A. Kondo, H. Takushi, K. Hayashi and O. Hashimoto, Thin wave absorber based on rubber sheet combined with flaky magnetic powder for electronic toll collection, Microwave Opt. Technol. Lett. 48, 1909 (2006).

${ }^{2}$ Y. Zhou, S. Jin, G. Qiu and M. Yang, Preparation of ultrafine nickel powder by polyol method and its oxidation product, Mater. Sci. Eng. B 122, 222 (2005).

${ }^{3}$ S.-S. Kim, S.-T. Kim, J.-M. Ahn and K.-H. Kim, Magnetic and microwave absorbing properties of Co-Fe thin films plated on hollow ceramic microspheres of low density, J. Magn. Magn. Mater. 271, 39 (2004).

${ }^{4}$ M.-G. Han, Y. Ou and L.-J. Deng, Microwave absorption properties of double-layer absorbers made of NiCoZn ferrites and hollow glass microspheres electrolessplated with FeCoNiB, $J$. Magn. Magn. Mater. 321, 1125 (2009).

${ }^{5}$ L.-Y. Li, Z.-Z. Zhang, F.-X. Zhao and F.-L. Shou, Preparation of Ultrafine nickel powder by high-energy ball milling, Surf. Technol. 41, 74 (2012).

${ }^{6}$ Y. Wang, L. Zhu and Y.-F. Fan, Preparation of Ni nano-particles by autocontinuous wire electrical explosion, China Powder Sci. Technol. 20, 52 (2014).

${ }^{7}$ L. Li, J.-H. Du, G.-Y. Gan, J.-K. Yan, J.-M. Zhang, Y.-C. Liu and J.-H. Yi, Study on preparation technology of nickel powder with liquid phase reduction method, Rare Metal Mater. Eng. 44, 0036 (2015).

${ }^{8}$ G.-Y. Huang, S.-M. Xu, G. Xu, L.-Y. Li and S. Z. Chen, Preparation and characterization of mono-disperse spherical nickel particles, Chin. J. Process Eng. 7, 1126 (2007).
${ }^{9}$ Q.-H. Huang, B.-S. Zhang, D.-M. Tang, Y. Yi, X.-G. Li and G.-B. $\mathrm{Ji}$, Synthesis and characteristics of graphene-Fe@ $\mathrm{Fe}_{3} \mathrm{O}_{4}$ nanocomposites materials, Chin. J. Inorg. Chem. 28, 2076 (2012).

${ }^{10}$ Z.-H. Yang, Z.-W. Li, L.-H. Yu, Y.-H. Yang and Z.-C. Xu, Achieving high performance electromagnetic wave attenuation: A rational design of silica coated mesoporous iron microcubes, $J$. Mater. Chem. C 2, 7583 (2014).

${ }^{11}$ X.-H. Chen, F.-C. Meng, Z.-W. Zhou, X. Tian, L.-M. Shan, S.-B. Zhu, X.-L. Xu, M. Jiang, L. Wang, D. Hui, Y. Wang, J. Lu and J.-H. Gou, One-step synthesis of graphene/polyaniline hybrids by in situ intercalation polymerization and their electromagnetic properties, Nanoscale 6, 8140 (2014).

${ }^{12}$ G.-H. Pan, J. Zhu, S.-L. Ma, G.-B. Sun and X.-J. Yang, Enhancing the electromagnetic performance of Co through the phase-controlled synthesis of hexagonal and cubic Co nanocrystals grown on graphene, ACS Appl. Mater. Interf. 5, 12716 (2013).

${ }^{13}$ Z.-L. Hou, M. Zhang, L.-B. Kong, H.-M. Fang, Z.-J. Li, H.-F. Zhou, H.-B. Jin and M.-S. Cao, Microwave permittivity and permeability experiments in high-loss dielectrics: Caution with implicit Fabry-Pérot resonance for negative imaginary permeability, Appl. Phys. Lett. 103, 162905 (2013).

${ }^{14}$ L.-J. Deng and M.-G. Han, Microwave absorbing performances of multiwalled carbon nanotube composites with negative permeability, Appl. Phys. Lett. 91, 023119 (2007).

${ }^{15}$ Y.-J. Chen, F. Zhang, G.-G. Zhao, X.-Y. Fang, H.-B. Jin, P. Gao, C.-L. Zhu, M.-S. Cao and G. Xiao, Synthesis, multi-nonlinear dielectric resonance, and excellent electromagnetic absorption characteristics of $\mathrm{Fe}_{3} \mathrm{O}_{4} / \mathrm{ZnO}$ core/shell nanorods, J. Phys. Chem. C 114, 9239 (2010). 